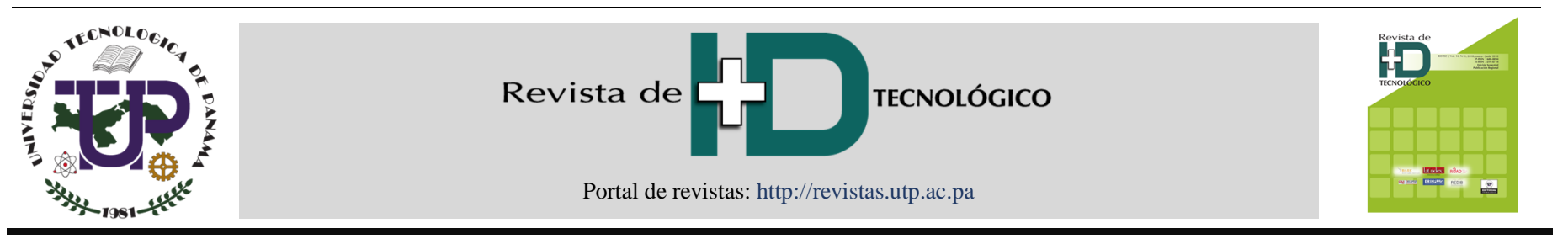

\title{
Estudio de percepción social en las vivencias postdesastre, para determinar el nivel de resiliencia y afectación comunitaria en el distrito de La Chorrera, provincia de Panamá Oeste
}

\section{Social perception study in the experiences postdisaster, to determine the community resilience and affectation level in La Chorrera, Panama Oeste}

\author{
Marta Moreno ${ }^{1 *}$, Humberto Álvarez ${ }^{1}$, Angelo Chacón ${ }^{3}$ \\ ${ }^{*}, 2$ Facultad de Ingeniería Industrial, Universidad Tecnológica de Panamá, Panamá \\ ${ }^{3}$ Licenciatura en Ingeniería Electromecánica, Universidad Tecnológica de Panamá, Panamá \\ *Autor de correspondencia: marta.moreno@utp.ac.pa
}

\begin{abstract}
RESUMEN- La evaluación concreta de la actitud de una población ante el riesgo y con ello el conocimiento es un factor básico en la valoración de su vulnerabilidad general, así como la búsqueda de las bases imprescindibles para poder dotarla de nuevas capacidades de defensa, exige un amplio trabajo de campo de índole interdisciplinar y, en particular la aplicación de metodologías cualitativas orientadas a la evaluación de experiencias comunitarias encuestas y entrevistas. Por lo tanto parece necesario recabar una información más amplia, que incluya el conocimiento de los problemas generales del grupo y el papel relativo que dentro de estos asignan a la existencia de riesgo; la evaluación que se hace, por parte de los afectados potenciales, de las acciones que emanan de los distintos niveles de la administración, acompañada por la descripción y valoración de su propia acción individual, familiar o de unidad de convivencia; también, por último, conocer el estado de opinión respecto a actuaciones que se estiman pertinentes y la valoración de propuestas ajenas, en el caso que existan. Este artículo presenta un estudio de percepción social en la gestión del riesgo de inundación en un área del distrito de La Chorrera, provincia de Panamá Oeste, que permitirá proponer un plan de acción para elevar y fortalecer la resiliencia comunitaria en este sector, y que el mismo pueda ser implementado en otras áreas de la República de Panamá.
\end{abstract}

Palabras clave-Gestión de riesgo, percepción social, resiliencia comunitaria.

\begin{abstract}
The assessment of the attitude of a population at risk and thus the knowledge of a basic factor in assessing their overall vulnerability, and the search for the essential foundation to provide it with new defense capabilities requires extensive work field of interdisciplinary nature and in particular the application of qualitative methodologies aimed at assessing community experiences surveys and interviews. It therefore seems necessary to obtain more comprehensive information, including knowledge of the general problems of the group and the relative role within these assigned to the existence of risk; the assessment made, by the potentially affected, actions emanating from the different levels of government, accompanied by the description and assessment of their own individual, family or household unit action; also, finally, to know the state of mind about actions that are considered relevant and evaluation of proposals outsiders, if any. This paper proposes Social Perception study on Flood Risk Management in an area of the district of La Chorrera, Panama, Panama Oeste, which will propose an action plan to increase and strengthen community resilience in this sector, and the same can be implemented in other areas of the Republic of Panama.
\end{abstract}

Keywords-Risk management, social perception, community resilience.

\section{Introducción}

Hay evidencia creciente de que los fenómenos meteorológicos extremos a corto plazo (por ejemplo, lluvias excesivas, calor, viento) son cada vez más frecuentes a nivel mundial, lo que podría provocar efectos negativos (es decir, inundaciones, sequías) y poner en peligro el ecosistema terrestre a largo plazo funcionando y estos aumentos son más evidentes en Norteamérica y Europa en comparación con otros países ubicados en el hemisferio sur, por ejemplo invierno de 2013-2014 vio niveles excepcionales de lluvia en el Reino Unido conduciendo a inundaciones extremas y prolongadas en muchas áreas bajas con tierras agrícolas que permanecen bajo el agua hasta tres meses y eventos similares han ocurrido en otros países como Estados Unidos en 2011, 2013 y 2014 y quizás el impacto más obvio de las inundaciones prolongadas en los campos agrícolas es el daño a los cultivos donde el suelo se

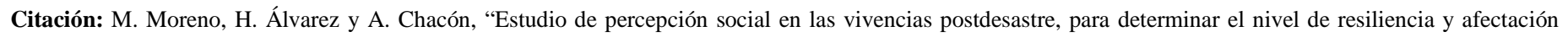
comunitaria en el distrito de La Chorrera, provincia de Panamá Oeste,” Revista de I+D Tecnológico, vol. 16, no. 1, pp. (90-103), 2020.

Tipo de artículo: Original. Recibido: 19 julio de 2019. Recibido con correcciones: 19 julio de 2019. Aceptado: 2 diciembre de 2019.

DOI.

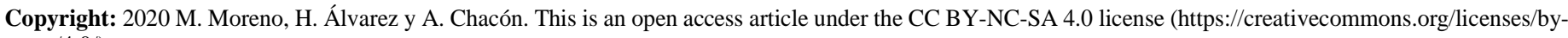
nc-sa/4.0/). 
convierte anaeróbico cuando está saturado de agua, y esto tiene efectos casi inmediatos en la vegetación. Dentro de las $48 \mathrm{~h}$, las plantas comienzan a sufrir privación de oxígeno, lo que provoca una reducción significativa en las tasas de absorción de nutrientes, inhibiendo crecimiento vegetal tanto por encima como por debajo del suelo, por lo tanto podría decirse que lo importante del concepto resiliencia en cualquier contexto es que define la capacidad de enfrentamiento a los cambios y la vuelta a la situación inicial, que por lo general implica adaptación y aprovechamiento incluso creación de mecanismos de superación [1].

Debido a la escasez de inundaciones extremas, se sabe relativamente poco del impacto a largo plazo de inundaciones prolongadas y posterior recuperación. Teniendo en cuenta que se prevé que experimentemos inundaciones más extremas en el futuro, es imperativo que comprendamos estos impactos y, lo que es más importante, cómo mitigar y aliviar el daño que puedan causar. La percepción del riesgo se relaciona con una construcción de pensamiento humano, siendo un resultado social y distinto de acuerdo con los contextos experimentados por individuos o grupos que generan múltiples interpretaciones del evento.

La percepción del riesgo es una herramienta para los servicios de protección civil y otras entidades de seguridad en los procesos de decisiones y acciones, la perspectiva social del riesgo es, sin duda, una importante herramienta en su gestión. La valoración de la percepción social del riesgo de inundación requiere un análisis integrado de los dos sistemas que lo integran, natural (peligrosidad) y el humano (exposición y vulnerabilidad). Hay distintos enfoques sobre la percepción, uno de ellos es el que se refiere a la forma en que un individuo interpreta y valora los posibles efectos y peligros de un riesgo. La percepción social de los episodios naturales otorga grado al impacto que causa un hecho natural de rango extraordinario.

Prevención de inundaciones: aquí se define como la prevención de ríos. Inundaciones, ha sido durante mucho tiempo una ideología universal. Se ha tomado para siempre que no se produzcan inundaciones en los centros urbanos o el desarrollo urbano se verá gravemente impedido. La prevención de inundaciones se logra en gran medida a través de la infraestructura de control de inundaciones (FCI por sus siglas en inglés) que utiliza medidas estructurales, como la canalización, diques, presas y desvío de inundaciones, para modificar la geomorfología e hidrología del río. A menudo retratado como un ejercicio técnico, la FCI está políticamente cargada y es moralmente significativa porque puede conferir seguridad contra inundaciones para algunos a costa de otros. Por ejemplo, FCI puede empeorar el riesgo de inundación para otros, y mientras esta inundación el riesgo es involuntario, es una imposición previsible. Sin embargo, relativamente poco ha sido discutido cómo las medidas de prevención de inundaciones, como intervenciones para brindar seguridad a ciertos grupos, podrían terminar comprometiendo el bienestar de otros [2].

En lo referido a la gestión de las inundaciones, esto se traduce en facilitar la inclusión de todos los potenciales afectados o interesados en una decisión, ya que los que se ven afectados por una decisión tienen el derecho a participar en el proceso de elección de esa decisión y sabemos que el riesgo de inundación es el resultado de las interacciones entre un peligro natural o antropogénico y las condiciones de vulnerabilidad de la sociedad. Sin embargo, no podemos olvidar que este concepto de riesgo también está configurado por la percepción social que existe de él. La noción sobre la percepción del riesgo de inundación está forjada por los juicios intuitivos que individuos o grupos de individuos hacen al respeto, utilizando para ello información limitada o incierta [2].

En la lista de amenazas naturales más frecuentes, las inundaciones vienen en primer lugar. Aun así, las comunidades urbanas no siempre están dispuestas a aceptar la inundación y hacer frente a las consecuencias.

El nivel de daños causados por las inundaciones y los eventos, el aumento y la función de las medidas estructurales existentes no proporcionan una protección aceptable.

La vulnerabilidad de las comunidades urbanas cobra importancia en el proceso de análisis de posibles estrategias para reducción de riesgos de inundaciones. Por definición, la vulnerabilidad es la medida en que un sistema es susceptible a inundaciones debido a exposición, una perturbación, junto con su capacidad (o incapacidad) para hacer frente, recuperarse o adaptarse, en consecuencia, el concepto de resiliencia está asumiendo un papel principal en este proceso debido al enfoque que está definiendo nivel de perturbación del sistema en su conjunto para mantenerse estable durante y después de la inundación. Aquí, bajo el sistema tanto físico y los componentes sociales están en juego. El término de resiliencia a menudo se deja abierto al debate y no tiene un general o definición consensual, aunque se utiliza cada vez más en la gestión integrada del drenaje urbano, las diversas interpretaciones de resiliencia 
reflejan la complejidad de este concepto. Esto dificulta la implementación de resiliencia en la gestión integral del drenaje urbano.

La discusión sobre la resiliencia debe subrayar el término resiliencia especificada con las siguientes preguntas: resiliente de ¿qué? y hasta que nivel? Estamos presentando resiliencia específica en este documento. La resistencia a las inundaciones define nivel aceptable de inundación que el sistema urbano puede tolerar, el sistema puede funcionar durante y después de la inundación [2].

Esto significa que es posible afirmar que el sistema urbano es resistente a los procesos de inundación. Hasta cierto nivel, los sistemas urbanos resistentes y las comunidades urbanas, deben tener la capacidad de aceptar, resistir, recuperarse y aprender de la capacidad de los sistemas urbanos y las comunidades, donde la misma se mejora en cada parte de la gestión del riesgo de inundaciones, cubriendo acciones relacionadas con la preparación, respuesta y recuperación. Dentro de esta investigación los cinco elementos de gestión del riesgo de inundación se desarrollan: reflejo, alivio, resistencia, respuesta y recuperación. Estos nuevos elementos donde, por lo tanto, estas nociones varían en la sociedad de acuerdo a diversos factores, como serían sus distintos niveles de conocimiento sobre el peligro (experiencias vividas en relación a anteriores episodios de inundación, nivel de educación e información recibida, etc.) o su ubicación geográfica y composición demográfica respecto a muchos otros.

En los últimos años, la percepción de riesgo se ha convertido en un tema importante para los tomadores de decisión relacionados con la gestión de riesgos, en la medida que su conocimiento dirige el desarrollo de estrategias de mitigación eficaz y eficiente. La percepción del riesgo es una herramienta para los servicios de protección civil y otras entidades de seguridad en los procesos de decisiones y acciones [3].

Es así como comprende que la inclusión de la comunidad en un enfoque reformado de la gobernanza del riesgo, propendería a aumentar una conciencia de relación con gobiernos locales y otras partes interesadas, posibilitando la elaboración de acciones en conjunto que brindan un bienestar colectivo. Un ejemplo de ello son las comunidades urbanas organizadas y las redes conjuntas entre gobierno y comunidades que se han desarrollado en las Filipinas.

Estas comunidades participan tanto en los procesos de identificación y priorización de las ayudas post catástrofes como también en el destino y utilización de los materiales entregados para vivienda y otros usos [3].

\section{Revisión del estado del arte}

Según la bibliografía revisada, existe en Panamá, el Marco Nacional de Recuperación de Panamá, en unos de sus contextos en respuesta a esta situación, los países de la región han dado importantes avances impulsados por el Centro de Coordinación para la Prevención de Desastres en América Central (CEPREDENAC): la formulación de la Política Centroamericana de Gestión Integral de Riesgos (PCGIR, 2010), los correspondientes planes nacionales, y la implementación de diversos programas de gestión de riesgos de desastres de carácter regional, nacional y local. Sin embargo, a pesar de estos avances se reconoce que existen todavía temas pendientes que abordar, entre ellos la gestión de procesos de recuperación.

En ese sentido, la Cumbre de Presidentes Centroamericanos de 2011 desarrollada, adoptó como prioridad la necesidad de fortalecer las capacidades institucionales y organizativas en la temática de recuperación postdesastre.

Es así que CEPREDENAC, con el apoyo técnico de la Oficina de Naciones Unidas para la Reducción de Riesgos de Desastres (UNISDR) y el financiamiento de la Agencia Australiana de Cooperación Internacional (AUS-AID), vienen apoyando la formulación de los marcos de recuperación en cada uno de los países de la región. Bajo esta condición, el presente Marco Nacional de Recuperación de Panamá se ha trazado el siguiente objetivo: "Orientar la intervención de instituciones públicas y privadas de manera organizada sectorial y territorialmente en procesos de recuperación postdesastre, atendiendo integralmente las necesidades y prioridades de la población de la zona afectada, y asegurando que no se regeneren las condiciones de riesgo y que se promuevan dinámicas más seguras de desarrollo".

Para ello, al interior de la Plataforma Nacional de Gestión Integral de Riesgo de Desastres (PNGIRD), se ha desarrollado un proceso participativo de análisis del problema y de las capacidades institucionales, de definición de tareas y de responsabilidades, y de diseño de mecanismos de coordinación para la recuperación de las zonas afectadas.

Bertilsson et tal definió objetivos concretos para alcanzar un estado de resiliencia a las inundaciones y evaluar su progreso, con el modelo matemático 
denominado S-FRESI en donde se consideraron diferentes variables y conjeturas de interés [4].

Sarmah et tal diseñaron una propuesta de un sistema de prevención y mitigación de inundaciones en la cuenca Bharalu ubicada en la rivera Bharalu en la India [5].

Tingsanchali et tal realizaron un estudio sobre las áreas vulnerables a inundaciones en Bangkok con el fin de sustentar un plan de resiliencia propuesto en conjunto con las autoridades [6].

Kuei-Hsien sustenta un paradigma alternativo de adaptación a las inundaciones, que trata de prevenir daños cuando se producen inundaciones y permite que las inundaciones ingresen a la ciudad y se basa en los trabajos de campo con las inundaciones en el delta del Mekong de Vietnam en dos aldeas, Vinh An y Ha Bao, donde la inundación es mayormente inofensiva debido a la experiencia de estas vivencias, las cuales aportan beneficios desarrollando una capacidad de respuesta ante inundaciones, de una manera más rápida enfocada hacia el área urbana [7].

Vazquéz-González et tal, estimaron el valor económico de la prevención de inundaciones provisto por los humedales costeros. Cuantificaron la pérdida de capacidad de retención de agua como función de pérdida de humedales y estimaron el costo económico de las inundaciones para los hogares conurbación VeracruzBoca del Río-Medellín en Veracruz, México donde se utilizó para evaluar estos parámetros durante las inundaciones causadas por el huracán Karl en 2010 [8].

Cai et tal formularon un estudio que utilizaron la dinámica de usó de la propiedad de simulación de un autómata celular para avanzar más en el enrutamiento por inundación, donde se consideraron varios parámetros de interés sobre la inundación y desarrollaron un modelo matemático basado en un autómata celular, además un caso numérico de propagación analizando esta inundación en un área de la parte baja del río Yangtze, utilizando los modelos de paso fijo y paso variable con el fin de enrutar las vías de las inundaciones [9].

Galderisi et tal, estudiaron el caso de la inundación ocurrida en la municipalidad de Benevento en Italia en donde se propusieron en el documento las áreas vulnerables y deficientes que están presentes en el área[10].

\subsection{Justificación}

En la última década, el Gobierno de Panamá realizó ocho declaraciones de emergencia de alcance nacional/regional. En diciembre de 2010, prolongadas precipitaciones provocaron serias inundaciones, impactaron seriamente al país y llevaron a declarar la emergencia nacional.

Estas inundaciones dejaron a gran parte de la ciudad de Panamá sin agua potable por semanas y obligaron al cierre del tránsito en el Canal de Panamá.

La asignación de fondos por sector para la respuesta y rehabilitación después de estas inundaciones superó la cifra de US\$ 149 millones.

Como podemos observar las inundaciones, vendavales y los deslizamientos son las amenazas naturales más frecuentes (ver figura 1).

En este sentido, inundaciones, vendavales y los deslizamientos agrupan el $79 \%$ de todos los eventos reportados en la base de datos del Sistema de Inventario de Efectos de Desastres (DesInventar entre 1929 y 2015), (ver figura 1).

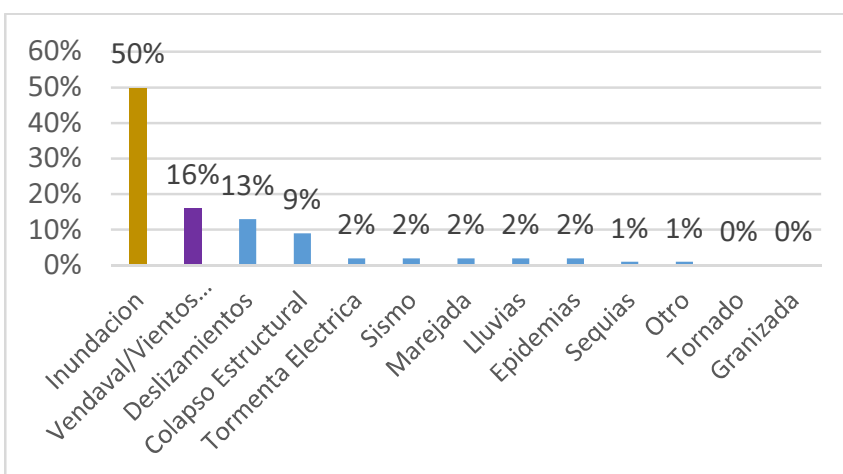

Figura 1. Estadísticas de amenazas naturales en Panamá (1929-2015).

Fuente: Base de datos del Sistema de Inventario de Efectos de Desastres (DESINVENTAR).

En este escenario, Panamá al igual que cualquier otro lugar del mundo no puede considerarse exento de tener que enfrentar situaciones indeseables más graves que las descritas en el párrafo anterior. Y en estos casos donde se han ameritado planes de contingencia por parte del gobierno, así como de otras organizaciones, han quedado descubiertos puntos críticos acerca de la organización y la comunicación entre los actores institucionales que participan en ellas, lo que afecta de manera directa el objetivo primordial de la logística humana, como es la recuperación de las personas afectadas.

Recientemente en el mes de septiembre de 2015, la dirección de Gestión Social de la Alcaldía de Panamá, a través de su equipo de trabajadoras sociales contabilizó mediante censo un aproximado de 25 casas con afectaciones en el corregimiento de Juan Díaz, provocadas por las inundaciones originadas por el 
torrencial aguacero del día jueves 10 de septiembre de 2015. (Redacción de TVN noticias 11/9/2015).

En el mes de octubre de 2015, 200 familias afectadas por inundaciones a causa de los oleajes en el Corregimiento de Puerto Caimito, Distrito de La Chorrera. (Redacción de TVN noticias 11/9/2015).

El motivo de elegir el caso de estudio en La Provincia de Panamá Oeste del Distrito de La Chorrera, ha sido por algunos escenarios de inundaciones en los periodos de 1996, 2003, 2006, 2012, afectando aproximadamente 3,699 personas, según informe suministrado por el Sistema Nacional de Protección Civil (SINAPROC) de Panamá Oeste, y algunas estadísticas recopiladas del Sistema de Inventario de Efectos de Desastre (DESINVENTAR).

Esta investigación tuvo como objetivo realizar un análisis de las vivencias postdesastre, a los afectados en las inundaciones del pasado 25 de noviembre de 2012, en el Distrito de Panamá Oeste, que ocasionaron aproximadamente 1,800 personas damnificadas de las áreas de los corregimientos de Barrio Colón, Barrio Balboa, El Coco, Mastranto Final, Puerto Caimito a través de un estudio de percepción social, que nos permita comparar el plan de recuperación postdesastre que actualmente existe en Panamá, y nos sirva como guía proponer un plan de acción, que fortalezca la resiliencia comunitaria en ese sector y sirva como referencia para otros sectores de la Provincia de Panamá y colaborar con la aplicación de las políticas que tienden al mejoramiento de la gestión de riesgo y el desarrollo sostenible en las áreas de vulnerabilidad a los desastres.

Daños causados por las inundaciones del pasado $25 \mathrm{de}$ noviembre de 2012, en el distrito de La Chorrera, (ver figuras 2, 3, 4).

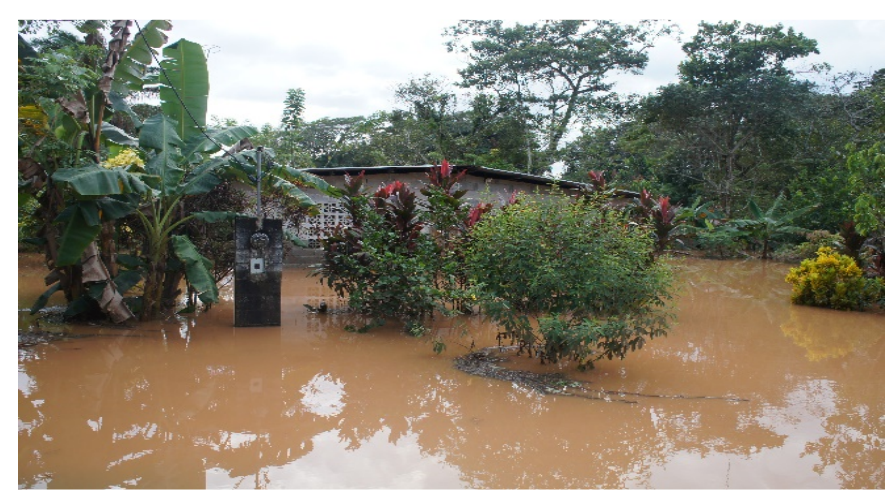

Figura 2. Fotografía de la inundación en Mastranto Final, La Chorrera - 2012

Fuente: Archivos del Municipio de La Chorrera.

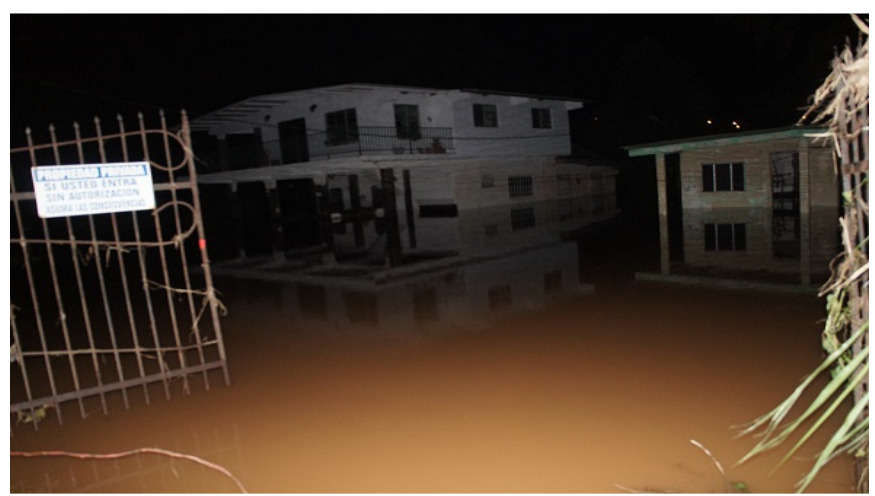

Figura 3. Fotografía de la inundación en el Trapichito, La Chorrera - 2012

Fuente: Archivos del Municipio de La Chorrera.

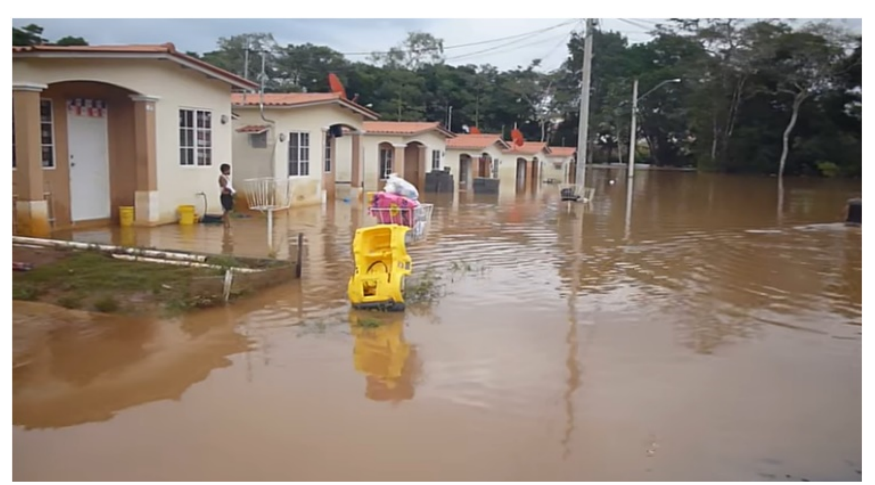

Figura 4. Fotografía de la inundación en La Barriada Los Flamingos, La Chorrera - 2012

Fuente: Archivos del Municipio de La Chorrera.

\section{Metodología}

\subsection{Descripción del área de estudio}

Panamá Oeste es una de las diez provincias de Panamá, creada el $1^{\circ}$ de enero de 2014 a partir de territorios segregados de la provincia de Panamá, ubicados al oeste del Canal de Panamá. Está conformado por cinco distritos: Arraiján, Capira, Chame, La Chorrera y San Carlos; y un total de 58 corregimientos. Su capital es el distrito de La Chorrera.

Limita al norte con la provincia de Colón, al sur con el océano Pacífico; al este con la provincia de Panamá y al oeste con la provincia de Coclé.

Cuenta con una extensión territorial de $2467.1 \mathrm{Km}^{2}$, para una población estimada en el 2014 de 518,013 habitantes y una densidad poblacional de 210 hab./Km ${ }^{2}$ siendo una de las más altas a nivel nacional. El Distrito de Arraiján el de mayor densidad (1538.7 hab./ $\left.\mathrm{Km}^{2}\right)$ y Capira el de menor densidad $\left(47.1 \mathrm{hab} . / \mathrm{Km}^{2}\right)$. (Ver figura $5)$. 


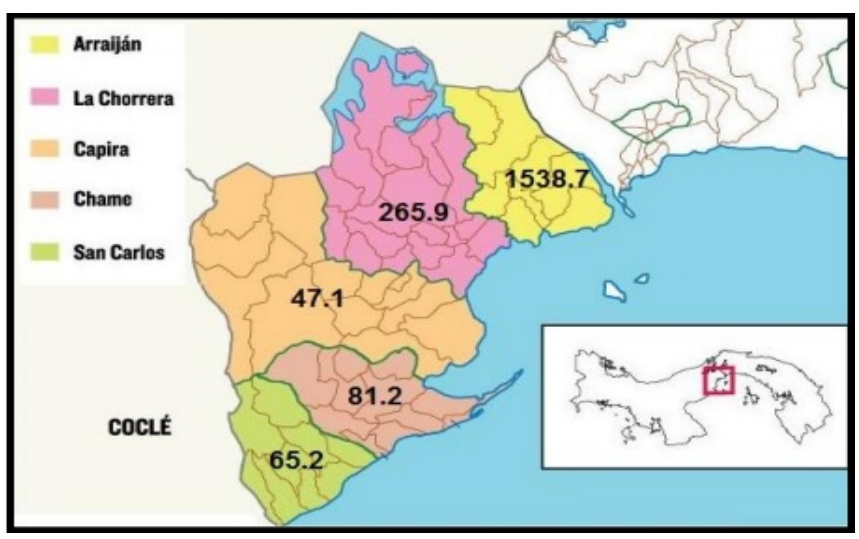

Figura 5. Densidad de población de Panamá Oeste, año 2014. Fuente: Sistema Regional de Salud Panamá Oeste. Departamento de Registros y Estadística de Salud.

\subsubsection{Localización de la cuenca}

La cuenca del río Caimito, cuenca 140, cuenta con una extensión de $509 \mathrm{~km}^{2}$ cuyo río principal es el río Caimito con una longitud de $72.83 \mathrm{Km}$ y un perímetro de 185.75 Km. Sus afluentes más importantes son: el río Aguacate con sus afluentes río Cáceres, San Bernardino y Potrero, en el distrito de Arraiján. En el distrito de la Chorrera se tienen afluentes importantes como son: Río Congo, Río Caimitillo y Río Martín Sánchez, (ver figura 6).

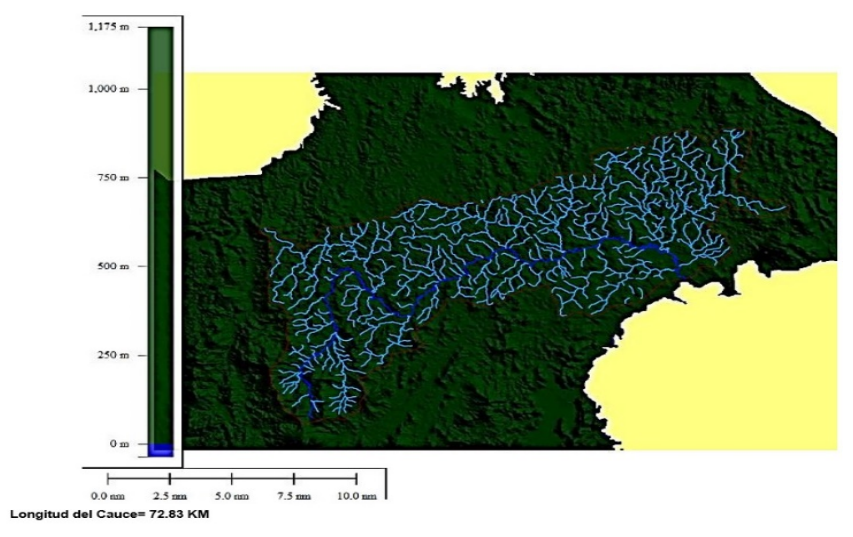

Figura 6. Cuenca del río Caimito, cuenca 140, cuenta con una extensión de 509 km2 cuyo río principal es el río Caimito con una longitud de $72.83 \mathrm{Km}$.

Fuente: Ing. Manuel Arcia.

En el distrito de Capira, donde tiene el nacimiento el Río Caimito, el principal afluente es el Río Caimitillo, lo demás lo constituyen quebradas de menor importancia. La cuenca del río Caimito ocupa gran parte de los distritos de La Chorrera, Arraiján y una pequeña porción del distrito de Capira. De los tres distritos, el de La Chorrera es el que tiene el mayor territorio, seguido del distrito de Arraiján y en menor proporción el distrito de Capira (ver figura 7).

El distrito de La Chorrera, a pesar de ocupar el mayor territorio de la cuenca del rio Caimito, la población se encuentra concentrada en la ciudad de La Chorrera. Aquí los corregimientos urbanos como Barrio Colón, Balboa y el Coco poseen la más alta densidad, alcanzando cifras que superan también los 1000 habitantes por kilómetro cuadrado (ver figura 6).

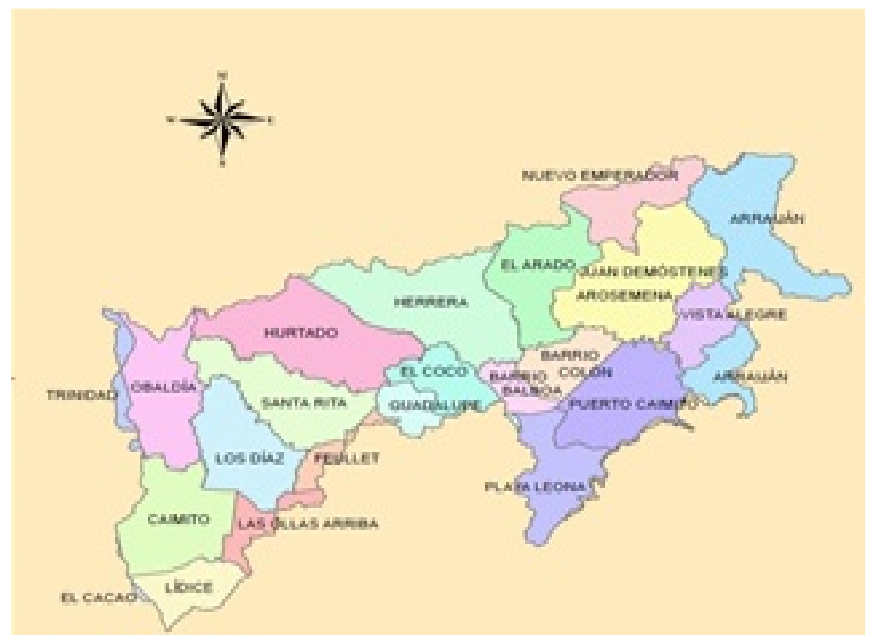

Figura 7. Corregimientos dentro de la cuenca del Río Caimito Fuente: Ing. Ricardo De León.

Según el artículo 23 de la ley 1 del 3 de febrero de 1994, establece lo siguiente: Queda prohibido el aprovechamiento forestal; el dañar o destruir árboles o arbustos en las zonas circundantes al nacimiento de cualquier cauce natural de agua, así como en las áreas adyacentes a lagos, lagunas, ríos y quebradas. Esta prohibición afectará una franja de bosques de la siguiente manera: Las áreas que bordean los ojos de agua que nacen en los cerros en un radio de doscientos (200) metros, y de cien (100) metros si nacen en terrenos planos. Para este análisis utilizamos la cobertura de poblados al cual se le establece un buffer con un corredor de 100 metros para verificar viviendas que se encuentran en esta área (ver figura 8). 


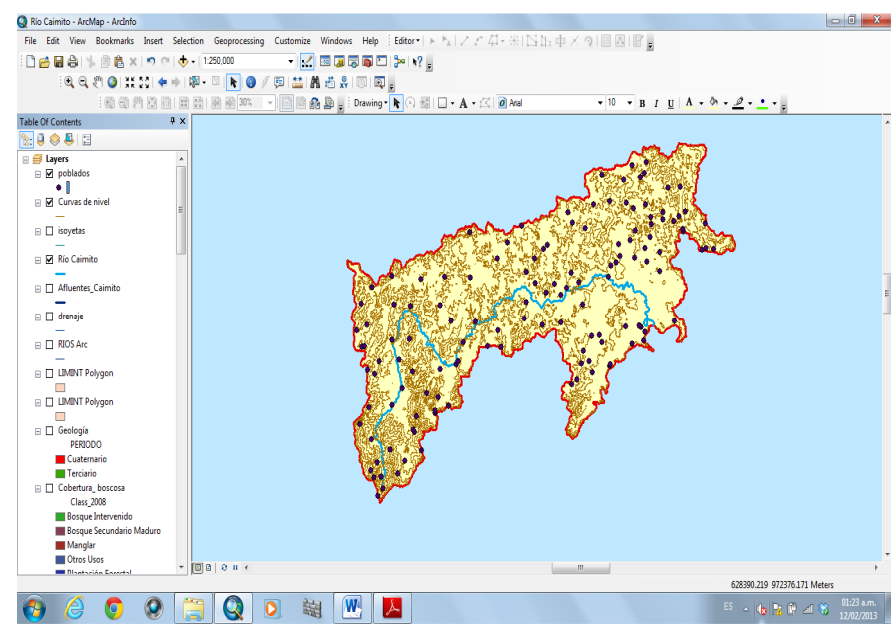

Figura 8. Poblados que están establecidos dentro de la cuenca del Río Caimito.

Fuente: Ing. Ricardo De León.

\subsubsection{Ubicación del área de estudio}

Mastranto final, ubicado en el corregimiento de Barrio Colón, distrito de La Chorrera, es uno de los sitios de estudio que fueron más afectados por esta inundación, con 114 casas afectadas y un total aproximadamente de 470 personas afectadas (ver figura 9).

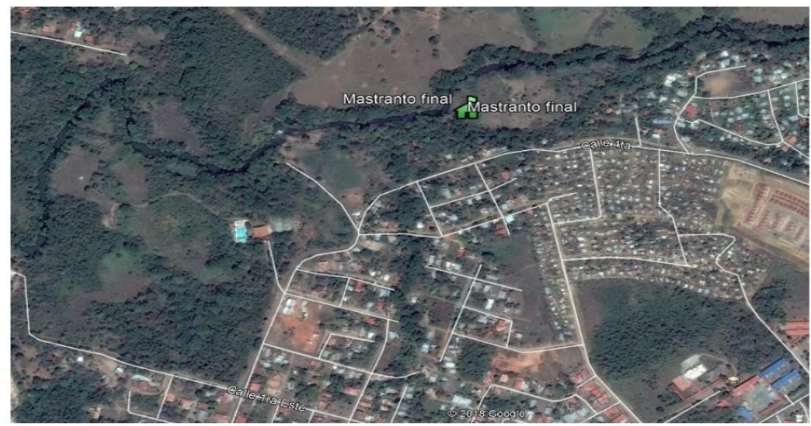

Figura 9. Ubicación satelital del área de Mastranto final Fuente: Lic. Italo Biancheri.

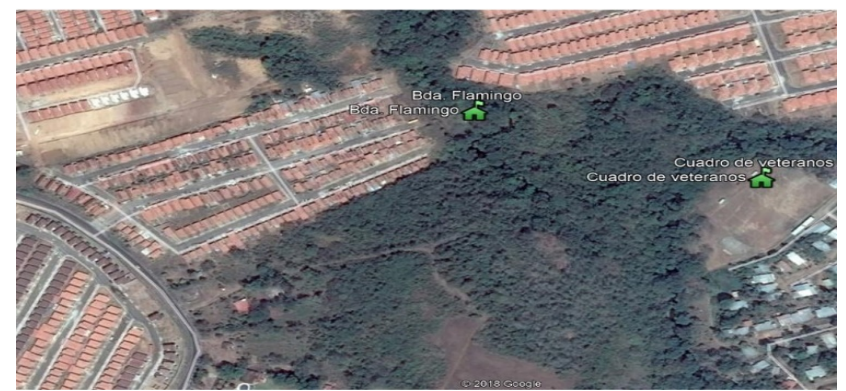

Figura 10. Ubicación satelital de la barriada Flamingo y el Cuadro de Veteranos ubicados en el corregimiento de Barrio Balboa, distrito El Trapichito.

Fuente: Lic. Italo Biancheri.

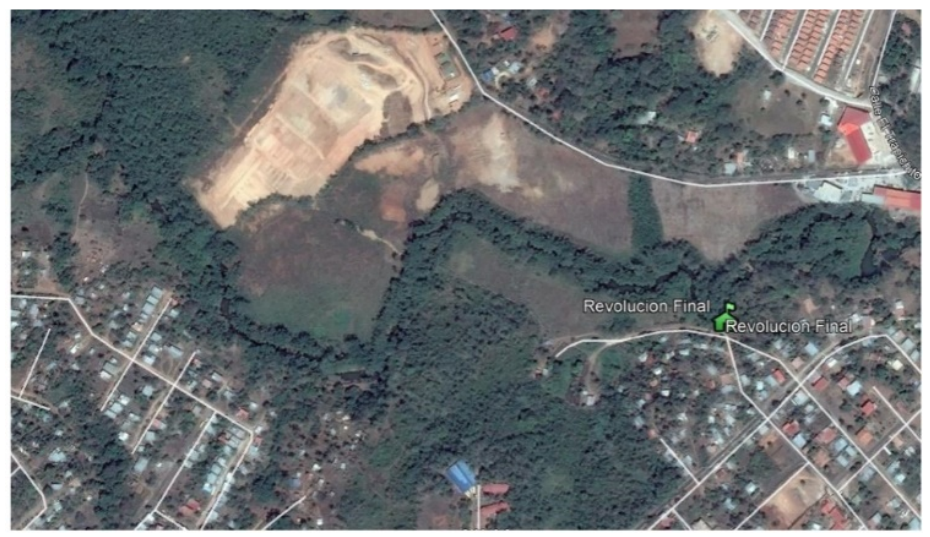

Figura 11. Ubicación satelital del área de Revolución Final ubicado en el distrito del El Trapichito, Corregimiento de Barrio Balboa.

Fuente: Lic. Italo Biancheri

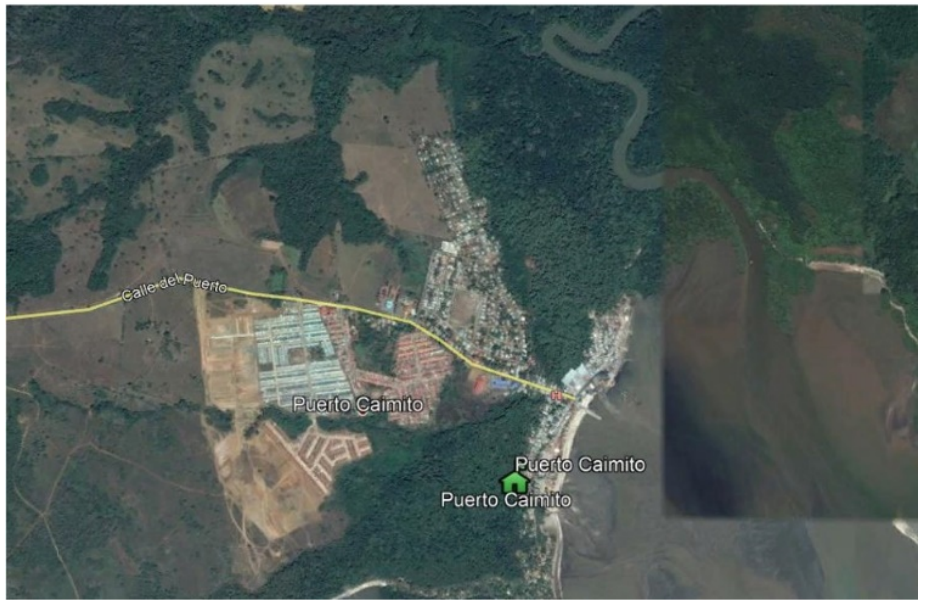

Figura 12. Ubicación del Puerto Caimito, distrito de Puerto Caimito.

Fuente: Lic. Italo Biancheri.

El estudio de la percepción del riesgo involucra un examen de conciencia de las personas, en base a sus emociones y comportamiento con respecto a las amenazas. Para este estudio se desarrolló una metodología mixta que combina aspectos cualitativos y cuantitativos y se aplican una técnica complementaria de recolección de datos: a) encuestas a los residentes de las zonas expuestas a las inundaciones.

El análisis de los aspectos perceptivos del riesgo se realizará por medio de encuestas. La finalidad de estas se centra de manera sistemática y ordenada en obtener información sobre variables que intervienen en la percepción de riesgos naturales. El cuestionario está formado por tres secciones: (1) Contexto general de la Comunidad (2); Descripción y evaluación del riesgo; (3) Preparación y respuesta ante el riesgo. Las encuestas 
intentarán determinar cuáles fueron las vivencias que tuvieron los moradores después del desastre y su percepción social que tuvo la comunidad afectada.

De acuerdo con la información recolectada la única estación disponible en la zona con datos de Temperatura y Precipitación corresponde a la Estación Caimito, ubicada en Panamá. Los datos disponibles para evaluar la precipitación se relacionan en la tabla 1.

Como se puede observar el año con menor precipitación fue el 2002 con solo $175,3 \mathrm{~mm}$ al año y el

Tabla 1. Precipitación Pluvial - Estación Caimito

\begin{tabular}{|c|c|c|c|c|c|c|c|c|c|c|c|c|c|}
\hline & \multicolumn{13}{|c|}{ Precipitación Pluvial en Centímetros } \\
\hline Panamá & 1994 & 1995 & 1996 & 1997 & 1998 & 1999 & 2000 & 2001 & 2002 & 2003 & 2004 & 2005 & Promedio \\
\hline Caimito & 1139,4 & 1591,1 & 1527,2 & 812,4 & 877,7 & 596,8 & 601,8 & 686,2 & 175,3 & 708,7 & 1399,9 & 708,7 & 902,1 \\
\hline
\end{tabular}

valor máximo se registró para el año 1995 con 1591,1 $\mathrm{mm}$ al año. Las precipitaciones en el área de estudio son generalmente de tipo convectivas y orográficas. Las corrientes marinas con altas temperaturas favorecen el calentamiento y evaporación de las aguas. A medida que el aire cargado de humedad, proveniente del Océano Pacífico, se traslada tierra adentro sobre la cuenca, las masas de aire se encuentran con las montañas ubicadas en la parte alta de las cuencas ocasionando precipitaciones.

El régimen pluvial o de precipitación del área de estudio pertenece a la vertiente del Pacifico, caracterizada por una notable precipitación entre los meses de mayo a noviembre. Las lluvias suelen ocurrir durante el día

\section{Elección del tipo de muestreo}

Hemos utilizado el muestreo probabilístico por conglomerado, ya que hay situaciones en los cuales la población está conformada por conglomerados que son grupos de individuos que tienen la particularidad de estar muy cercanos unos a otros, cuando establecer una lista de todos los individuos resulta muy difícil o cuando una selección aleatoria de estos implicara tener observaciones que podrían quedar muy distantes una de otras, lo que resultaría muy costoso, es posible seleccionar primero conglomerados, en forma aleatoria, y dentro de estos a los individuos de interés para el estudio. Si los individuos son heterogéneos dentro del conglomerado se observan varios o todos sus componentes, de lo contrario si son muy homogéneos basta con pocas observaciones.

\section{Preparación del marco de muestreo}

Actualmente se cuenta con una data en Excel de todos los afectados en las inundaciones del pasado $25 \mathrm{de}$ noviembre de 2012, en el Distrito de Panamá Oeste, que ocasionaron aproximadamente 1,800 personas damnificadas de las áreas de los corregimientos de Barrio Colón, Barrio Balboa, El Coco, Mastranto Final. (Sistema Nacional de Protección Civil de Panamá Oeste, 2012).

\section{Determinación del tamaño de la muestra}

Elegido el esquema de muestreo, ahora es necesario determinar aspectos referentes a la muestra, en particular su tamaño y su procedimiento de selección.

Como contamos con una data suministrada por el (Sistema Nacional de Protección Civil de Panamá Oeste, 2012), presente en la tabla 1. en donde nos indican la cantidad de personas damnificadas por vivienda, para que la muestra fuera más representativa se hizo el estudio a través de una muestra de todas las viviendas que fueron afectadas por la inundación el pasado 25 de noviembre de 2012, por sector, además de contener dicha muestra se hace una referencia en cuanto al margen de error de la encuesta.

\section{Cálculo del tamaño de la muestra conociendo el tamaño de la población}

La fórmula que utilizamos para calcular el tamaño de muestra cuando se conoce el tamaño de la población es la siguiente:

En donde,

$$
n=\frac{N Z_{a}^{2} p q}{d^{2}(N-1)+Z_{a}^{2} p q}
$$

$\mathrm{N}=$ tamaño de la población $(1,812)$

$\mathrm{Z}_{\mathrm{a}}=$ nivel de confianza, (1.96)

$\mathrm{p}=$ probabilidad de éxito, o proporción esperada $(0.05)$

$\mathrm{q}=$ probabilidad de fracaso $(0.5)$ 
$\mathrm{d}=$ precisión (Error máximo admisible en términos de proporción)

Nivel de confianza $=95 \%$;

Error de la medida $=1.309522 \%$;

Proporción esperada $=$ asumamos que puede ser próxima al 5\%; si no tuviese ninguna idea de dicha proporción utilizaríamos el valor $\mathrm{p}=0.5(50 \%)$ que maximiza el tamaño muestral.

A continuación, se muestra el cálculo para determinar el tamaño de la muestra de toda la encuesta.

$$
\begin{gathered}
n=\frac{(1812)(1.96)^{2}(0.05)(0.5)}{(0.01309522)^{2}(1812-1)+(1.96)^{2}(0.05)(0.5)} \\
n=428
\end{gathered}
$$

Tabla 2. Informe preliminar de las casas y personas afectadas en el año 2012 en las inundaciones en La Chorrera, provincia de Panamá Oeste

\begin{tabular}{|l|c|c|c|}
\hline \multicolumn{1}{|c|}{ Lugar } & $\begin{array}{c}\text { Casas } \\
\text { Afectada } \\
\text { S }\end{array}$ & $\begin{array}{c}\text { Cálculo del } \\
\text { tamaño de } \\
\text { la muestra }\end{array}$ & $\begin{array}{c}\text { Personas } \\
\text { Afectada } \\
\text { s }\end{array}$ \\
\hline La Goyita & 4 & $\mathbf{3}$ & 18 \\
\hline $\begin{array}{l}\text { Moisés } \\
\text { Castillo }\end{array}$ & 7 & $\mathbf{6}$ & 26 \\
\hline El Limón & 15 & $\mathbf{1 4}$ & 58 \\
\hline $\begin{array}{l}\text { Mastranto } \\
\text { Final }\end{array}$ & 114 & $\mathbf{1 0 3}$ & 470 \\
\hline El Campesino & 34 & $\mathbf{3 2}$ & 130 \\
\hline El Trapichito & 21 & $\mathbf{2 0}$ & 89 \\
\hline $\begin{array}{l}\text { Cuadro } \\
\text { Veterano }\end{array}$ & 4 & $\mathbf{3}$ & 14 \\
\hline $\begin{array}{l}\text { Puente } \\
\text { Velásquez }\end{array}$ & 21 & $\mathbf{2 0}$ & 87 \\
\hline $\begin{array}{l}\text { Revolución } \\
\text { Final }\end{array}$ & 60 & $\mathbf{5 6}$ & 268 \\
\hline $\begin{array}{l}\text { Naos La } \\
\text { Riviera }\end{array}$ & 32 & $\mathbf{3 1}$ & 129 \\
\hline $\begin{array}{l}\text { El Coco } \\
\text { Raudal No.2 }\end{array}$ & 7 & $\mathbf{6}$ & 27 \\
\hline Sta. Rita & 22 & $\mathbf{2 1}$ & 56 \\
\hline Obaldía & 13 & $\mathbf{1 2}$ & 56 \\
\hline Bda. Flamingo & 48 & $\mathbf{4 5}$ & 184 \\
\hline $\begin{array}{l}\text { Puerto } \\
\text { Caimito }\end{array}$ & 60 & $\mathbf{5 6}$ & 200 \\
\hline \multicolumn{1}{|c|}{ Total } & $\mathbf{4 6 2}$ & $\mathbf{4 2 8}$ & $\mathbf{1 8 1 2}$ \\
\hline
\end{tabular}

Se detallan los cálculos de la muestra de las viviendas afectadas por las inundaciones, en 15 lugares, del Distrito de La Chorrera, Provincia de Panamá Oeste.

Se encuestaron 428 viviendas afectadas, por las inundaciones para poder tener una confiabilidad del $95 \%$.

\subsection{Estudio de factibilidad}

Para poder resolver este modelo utilizamos el programas informáticos SPSS (es un programa estadístico informático muy usado en las ciencias sociales y las empresas de investigación de mercado. Originalmente SPSS fue creado como el acrónimo de Statistical Package for the Social Sciences, aunque también se ha referido como "Statistical Product and Service Solutions" (Pardo, A., \& Ruiz, M.A., 2002, p. 3). Sin embargo, en la actualidad la parte SPSS del nombre completo del software (IBM SPSS) no es acrónimo de nada.

\section{Resultados}

Una vez realizado el estudio y el análisis correspondiente, los resultados obtenidos los clasificamos de la siguiente manera. Los siguientes gráficos corresponden a la tabulación de las encuestas que han sido respondidas por los participantes. Los datos que se han extraído de estas encuestas, corresponden al contexto general de la comunidad, descripción y evaluación del riesgo y preparación y respuesta.

\section{Contexto general de la comunidad}

En relación a la pregunta cuál es su nivel de ingreso familiar mensual: en la figura 10, se señala que el $76 \%$ de los encuestados respondieron menos de 500.00, el $22 \%$ de 500.00 a 900.00 y el $1 \%$ de 1000.00 a 1500.00 . En la figura 11, muestra el tiempo que tienen las personas de vivir en las áreas inundables, pudimos observar que la mayoría de las personas en cada comunidad tiene más de 11 años de vivir en esa comunidad. En la figura 12, el $78 \%$ de los encuestados respondieron que tienen conocimiento de vivir en un área inundable, el 13\% contesto que No y el otro $10 \%$ contestó que no sabe. 


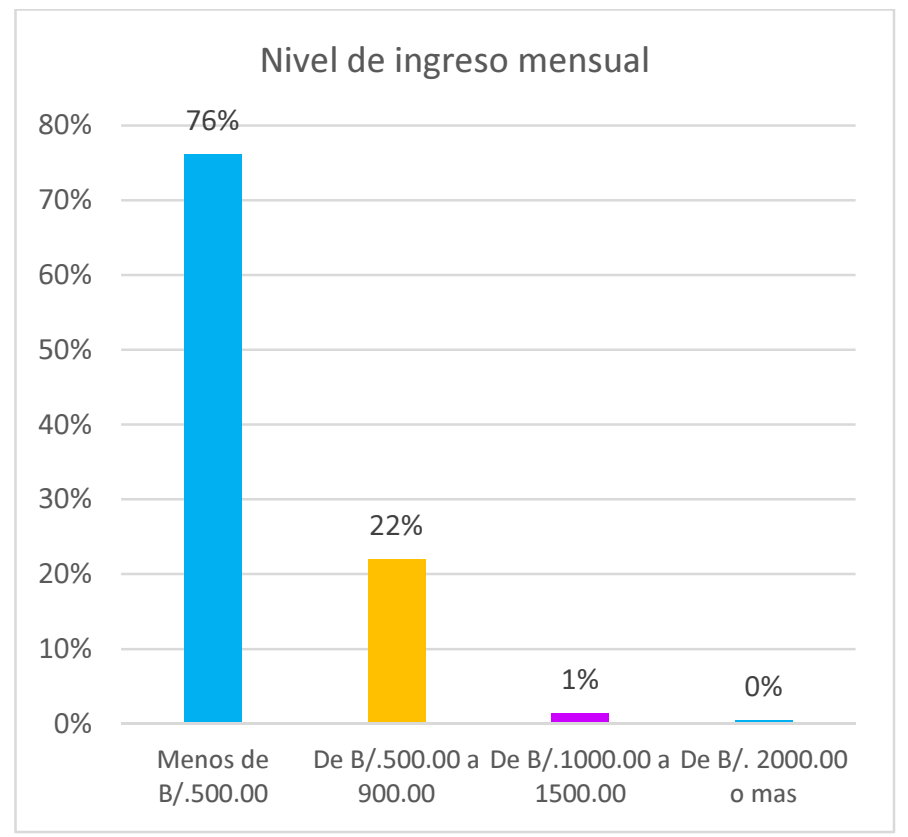

Figura 10. Nivel de Ingreso Mensual.

Fuente: Los autores.

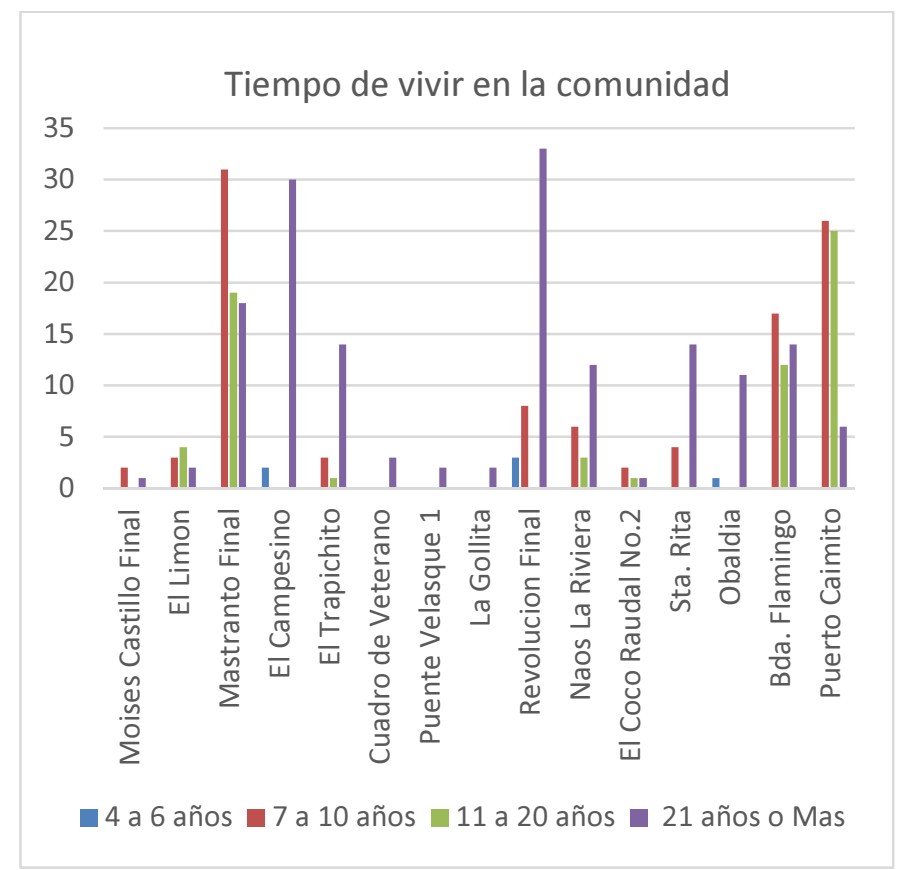

Figura 11. Tiempo de vivir en la comunidad.

Fuente: Los autores.

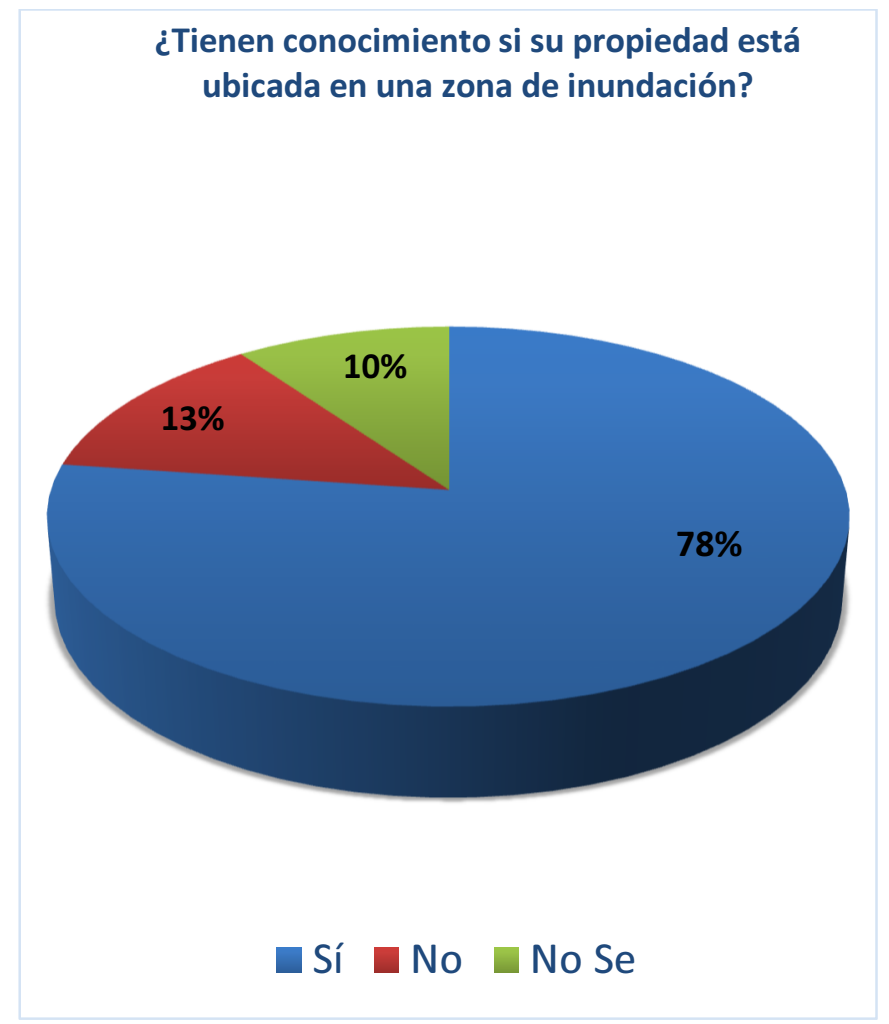

Figura 12. Tienen conocimiento si su propiedad está ubicada en una zona de inundación.

Fuente: Los autores.

\section{Descripción y Evaluación del Riesgo}

En relación a la pregunta cuál es su nivel de ingreso familiar mensual: En relación a la pregunta cuál fue el evento de esta inundación, en la figura 13 podemos observar que, en la mayoría de los moradores de La Goyita, Moisés Castillo, El Limón, Mastranto Final, El Campesino, El Trapichito, Cuadro Veterano, Puente Velásquez, Revolución Final, Naos La Riviera, El Coco Raudal No.2, Sta. Rita, Obaldía, y Bda. Flamingo respondieron que el evento de la inundación fue causado por un río, en excepto los moradores de Puerto Caimito manifestaron que el evento de la inundación, fue provocado por la costa. En cuanto a la pregunta cuál fue el nivel de la inundación, en la figura 14 muestra que la mayoría de las respuestas coincidieron en todas las comunidades en donde el nivel de la inundación alcanzo en su mayoría 0.5 a 1 metro en adelante y en la figura 15 el 56\% manifestaron que la frecuencia de inundación es de 2 veces al año, el $43 \%$ manifestaron que es de una vez al año el $1 \%$ más de 5 veces al año. 


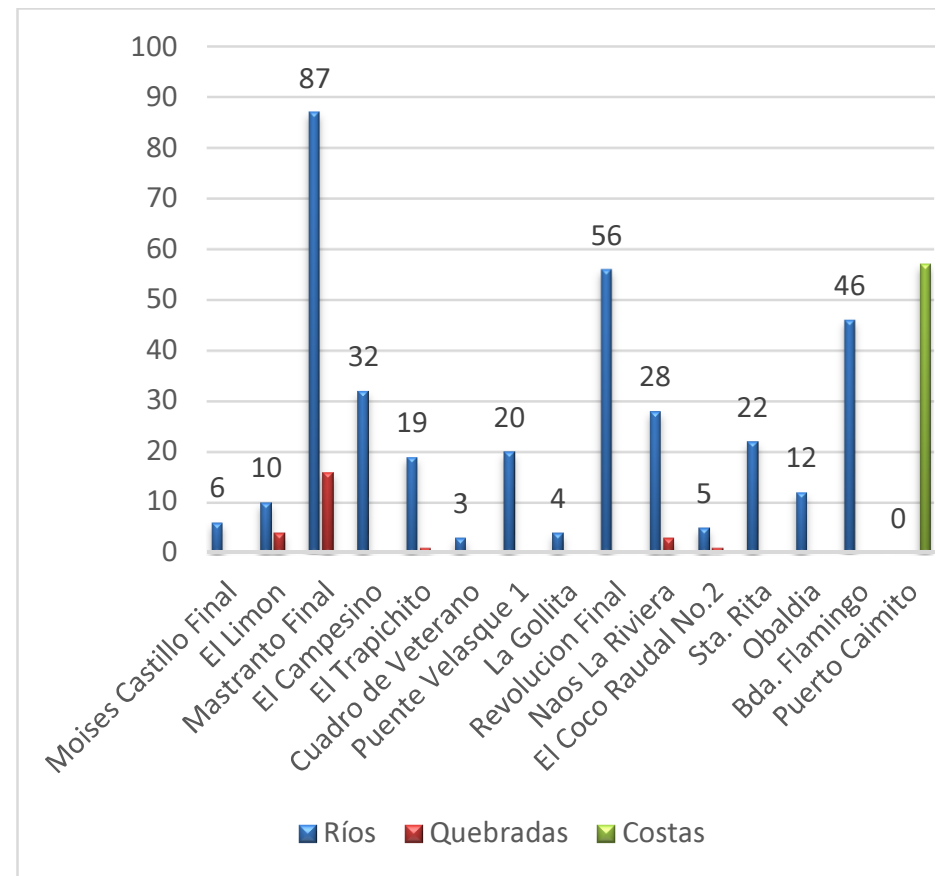

Figura 13. Lugares en donde se encuestó de dónde provino la inundación.

Fuente: Los autores.

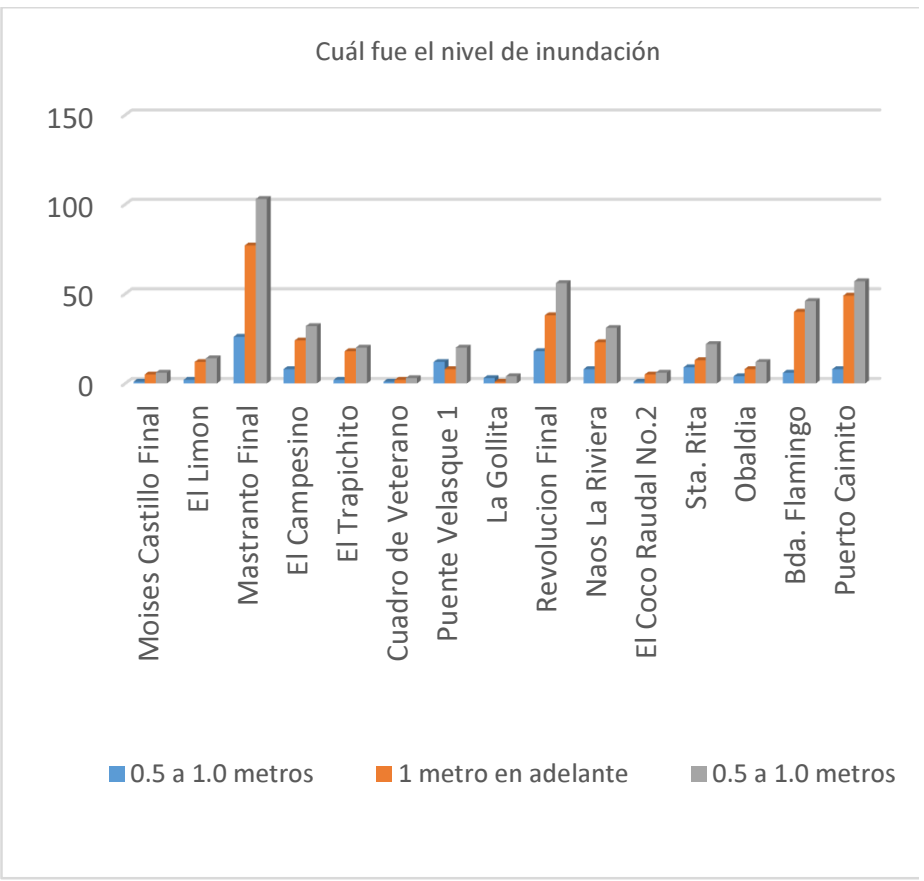

Figura 14. Encuesta en donde se preguntó hasta dónde fue el nivel de agua producto de la inundación.

Fuente: Los autores.

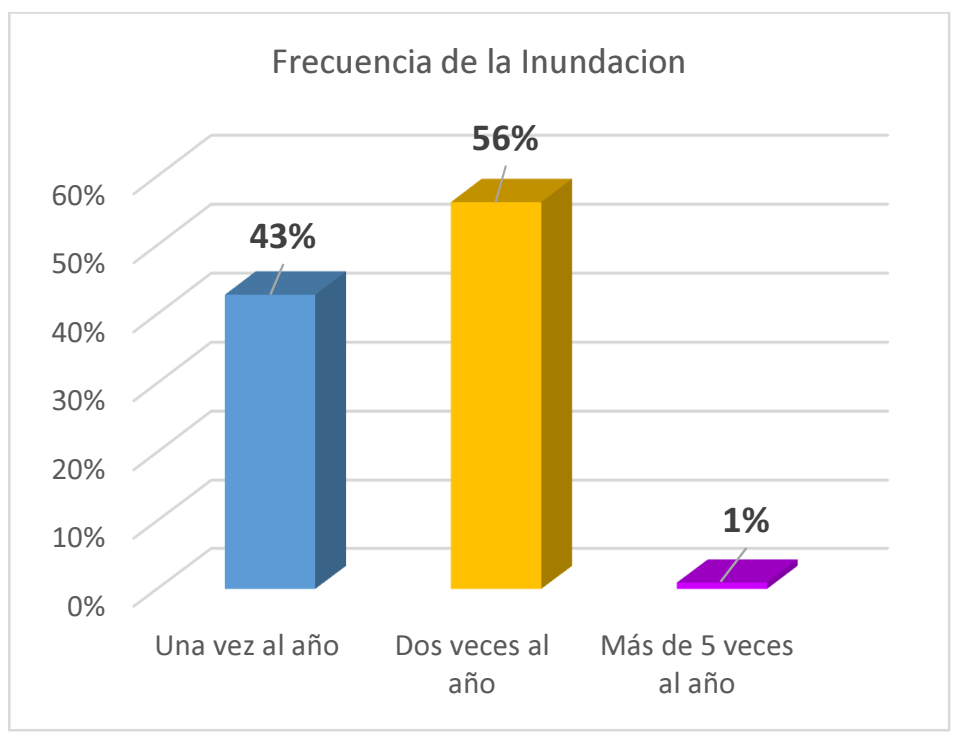

Figura 15. Encuesta en donde se determinó la frecuencia de las inundaciones.

Fuente: Los autores.

\section{Preparación y respuesta}

En la figura 16 el $56 \%$ de los moradores encuestados manifestaron que la ayuda recibida al momento de la inundación no fue nada oportuna, el 35\% manifestó que fue un poco oportuna, el $8 \%$ que fue oportuna y $0 \%$ muy oportuna.

Tiempo en que recibieron la ayuda

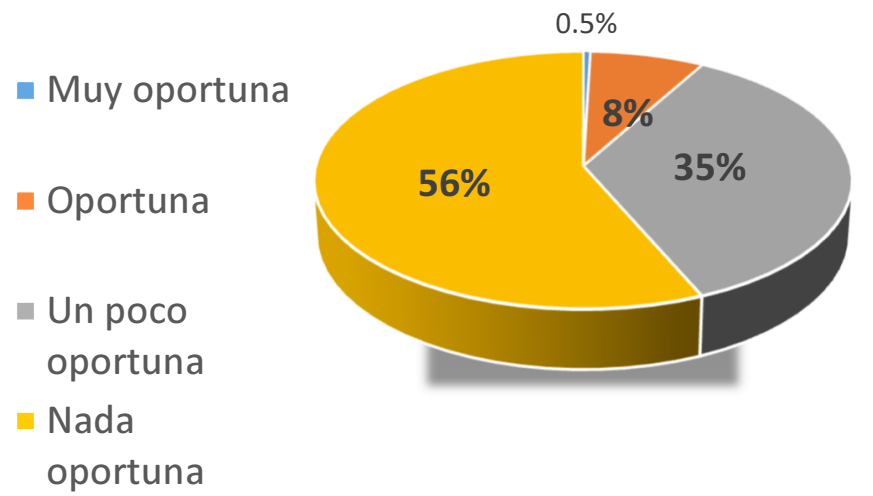

Figura 16. Encuesta en donde se realizó la pregunta del nivel de respuesta percibida por parte de las autoridades en el área del desastre.

Fuente: Los autores. 
En relación a la pregunta, "considera usted que las autoridades han tomado las medidas necesarias para minimizar los riesgos de inundación", en la figura 17 pudimos observar que el $87 \%$ de los moradores manifestaron que las autoridades No han tomado las medidas necesarias para minimizar esta inundación y el $13 \%$ manifestaron que Sí.

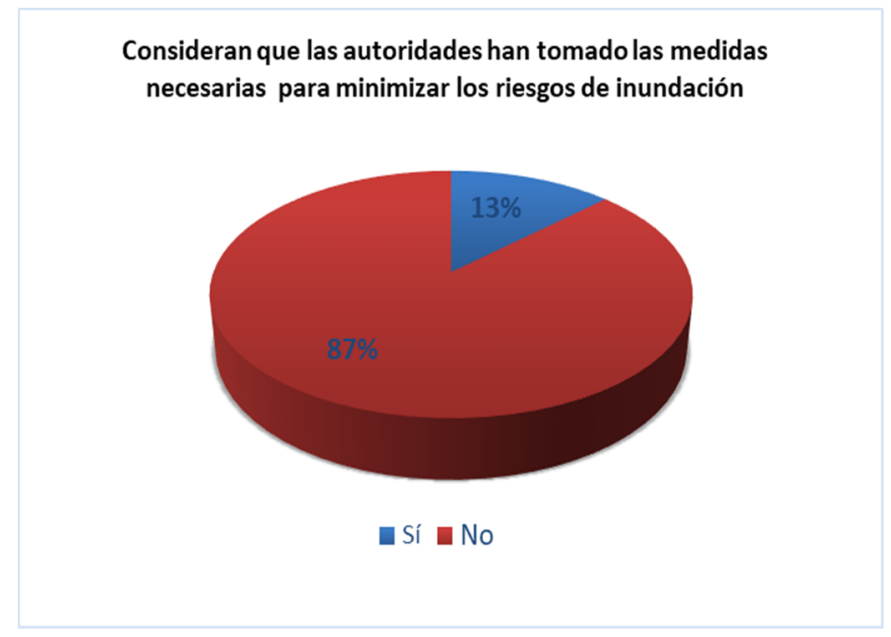

Figura 17. Encuesta en donde sustentaron si las autoridades tomaron medidas oportunas en el área del desastre.

Fuente: Los autores.

Como podemos observar en la figura 18, el $89 \%$ de los moradores respondieron que no se les ha capacitado para enfrentar las inundaciones, y el $11 \%$ restantes respondieron que sí. El resultado de esta encuesta expresa el desinterés por las autoridades sobre el tema de las inundaciones, la cual no hace algo al respecto para minimizar las mismas.

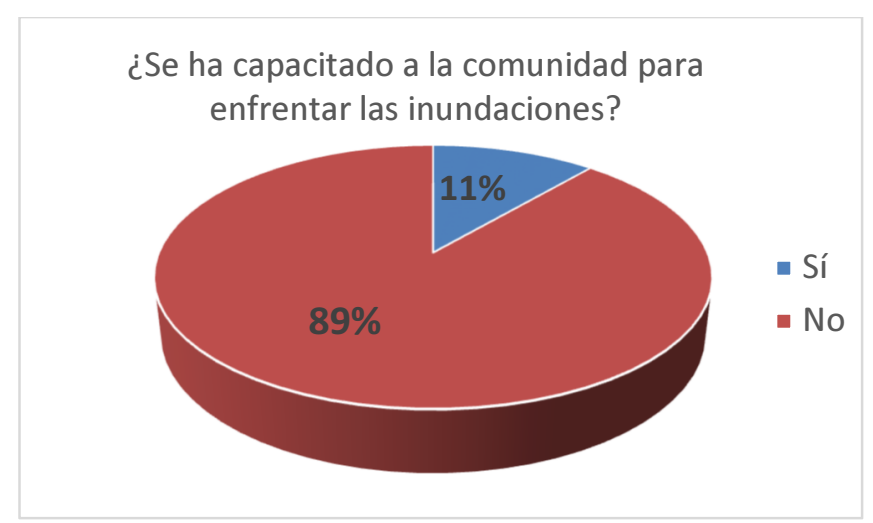

Figura 18. Encuesta en donde se determinó el nivel de capacitación de la comunidad.

Fuente: Los autores.
En cuanto al tipo de ayuda recibida en el momento del desastre en la figura 19 , el $96.3 \%$ de los moradores manifestaron haber recibido en su gran mayoría enseres.

\section{Tipo de ayuda que recibió al momento de la inundación}

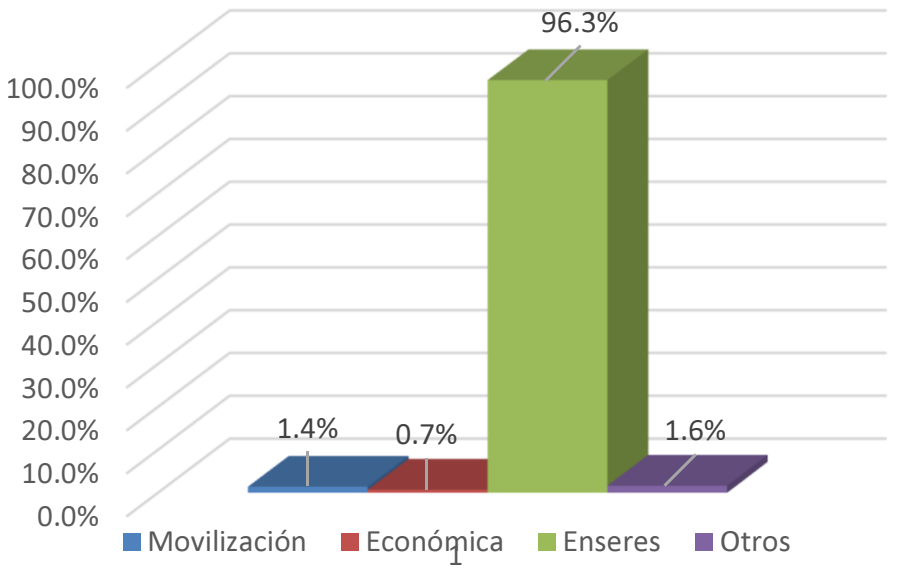

Figura 19. Encuesta en donde se preguntó acerca del tipo de ayuda recibida en el momento del desastre.

Fuente: Los autores.

¿Existe un sistema de alerta temprana, operativo en su comunidad?

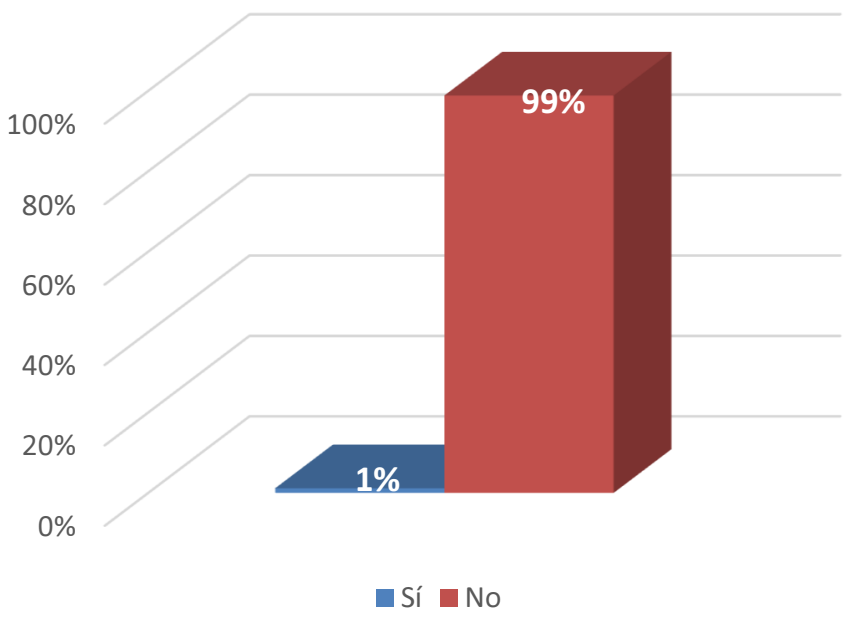

Figura 20. Encuesta sobre un sistema de alerta temprana en el área afectada.

Fuente: Los autores. 
En la figura 20 se sustenta que la falta de un sistema de alerta temprana en sus comunidades, los moradores tienen desconocimiento en cuanto el riesgo de una inundación inminente, tomando en cuenta el peligro de la situación para entonces inmediatamente realizar una evacuación oportuna ante el desastre, en este caso una inundación.

Tomando en cuenta todas las figuras anteriores, con relación a un análisis en función de las personas con respecto con la ayuda recibida, se consideró que la ayuda proporcionada por el personal del Sistema de Protección Civil fue de rescate, limpieza y suministros, tomando en cuenta que las condiciones del lugar no se propiciaron para una asistencia inmediata, debido a que la inundación bloqueó y dejó incomunicada las vías de tránsito habitual.

\section{Conclusión}

La percepción social de los riesgos naturales es producto de muchos factores como el resultado del universo social y grado de conocimiento de situaciones similares. Se ha observado en la muestra estudiada en el artículo que las personas que pasan por dificultades debido a efectos de fenómenos naturales, como por ejemplo las inundaciones, son más conscientes en relación a las acciones que debemos tomar respecto al medio ambiente. La experiencia con el riesgo, esto es, la vivencia de algún episodio extremo en fecha reciente, es el factor de mayor correlación con la percepción del riesgo, en la muestra estudiada.

Mediante una encuesta realizada como sujeto de estudio a los moradores de la cuenca del río Caimito, en donde se determinaron las siguientes deficiencias sustentadas por dichas personas:

- Deficiencia en la ayuda por parte de las autoridades.

- Deficiencia en la capacidad de hacer frente a las inundaciones por medio de capacitación.

- Deficiencia de un sistema de alerta temprana presente en el área afectada.

Dicho todo anterior se determinó que las personas en el área afectada no tienen capacitación ni conocimiento alguno sobre cómo hacer frente a las inundaciones, por lo tanto, se considera que no existe resiliencia alguna por parte de la población. Aunado a esto se expresó que la ayuda de las autoridades no fue nada oportuna, y tomando en cuenta eso se sustenta también que no existe un sistema de alerta temprana donde dichas personas no tienen una referencia o aviso cuando van a ocurrir algunos de estos desastres, poniendo en riesgo no solo la integridad física y la vida de esas personas sino también sus bienes.

\subsection{Recomendaciones}

Tabla 3. Esquema de acción postdesastre

\begin{tabular}{|c|c|c|}
\hline $\begin{array}{c}\text { Desastre/ } \\
\text { emergencia }\end{array}$ & \multicolumn{2}{|c|}{ Postdesastre } \\
\hline Respuesta & Recuperación & Desarrollo \\
\hline $\begin{array}{l}\text { Movilizar ayuda } \\
\text { financiera como } \\
\text { garantías y } \\
\text { créditos a largo } \\
\text { plazo }\end{array}$ & $\begin{array}{l}\text { Financiar programas } \\
\text { de rehabilitación y } \\
\text { de alimentos por } \\
\text { trabajo }\end{array}$ & $\begin{array}{l}\text { Incluir } \\
\text { actividades de } \\
\text { GRD en la } \\
\text { planificación del } \\
\text { desarrollo }\end{array}$ \\
\hline $\begin{array}{l}\text { Declarar } \\
\text { desastres y } \\
\text { estado de } \\
\text { emergencia } \\
\end{array}$ & $\begin{array}{l}\text { Establecer fondos } \\
\text { para emergencia y } \\
\text { recuperación }\end{array}$ & $\begin{array}{l}\text { Preparar código } \\
\text { de conducta para } \\
\text { desarrollo y } \\
\text { socorro } \\
\end{array}$ \\
\hline $\begin{array}{l}\text { Coordinar y } \\
\text { mediar } \\
\text { acciones entre } \\
\text { los niveles } \\
\text { locales } \\
\text { y nacionales }\end{array}$ & $\begin{array}{l}\text { Implementar } \\
\text { alimentos u otros } \\
\text { programas de } \\
\text { rehabilitación }\end{array}$ & $\begin{array}{l}\text { Proteger las } \\
\text { infraestructuras: } \\
\text { promover } \\
\text { tecnologías para } \\
\text { la reducción del } \\
\text { riesgo. }\end{array}$ \\
\hline $\begin{array}{l}\text { Ayudar en } \\
\text { evaluación } \\
\text { de necesidades } \\
\text { y distribución de } \\
\text { insumos } \\
\text { específicos por } \\
\text { sector }\end{array}$ & $\begin{array}{l}\text { Promover procesos } \\
\text { de recuperación } \\
\text { específicos por } \\
\text { sector }\end{array}$ & $\begin{array}{l}\text { Desarrollar } \\
\text { tecnologías para } \\
\text { la reducción de } \\
\text { riegos. }\end{array}$ \\
\hline
\end{tabular}

Mediante estos diagramas conceptuales se expresa de manera sencilla los posibles planes de acción y planificación propuesto para las autoridades en conjunto con la población donde GRD es Gestión de Riesgo de Desastres.

Tomando en cuenta este documento científico se recomienda un estudio a fondo para predecir las áreas aledañas de riesgo y generar un mapa de riesgo, además de un plan de acción en donde se requiere que las autoridades tengan pleno conocimiento para así brindar una ayuda rápida y oportuna a los damnificados por lo tanto se va a sustentar próximamente un modelo matemático y simulación de estas en un próximo artículo.

Por lo cual en el próximo artículo en base a toda la información recabada se planea hacer una simulación hídrica del área para generar un mapa de riesgo de toda 
la cuenca sustentada por un modelo matemático en las ecuaciones de Navier-Stokes enfocadas en las inundaciones, luego de realizar lo anterior se planea hacer un plan de planificación incorporando un sistema de alerta temprana en la ubicación de estudio.

Según la tabla 2 se recomienda en base a el desastre ocurrido implementar un sistema de alerta temprana además de realizar un plan riguroso de capacitación a la población para reducir los riesgos de no solamente de perder vidas sino reducir las pérdidas materiales, ya que la gran mayoría de los moradores del área de la cuenca no cuentan con salarios suficientes para recuperar lo perdido.

\section{Referencias}

[1] R. J. Harvey, D. R. Chadwick, A. R. Sánchez-Rodríguez, y D. L. Jones, «Agroecosystem resilience in response to extreme winter flooding», Agric. Ecosyst. Environ., vol. 279, pp. 1-13, jul. 2019.

[2] K.-H. Liao, J. K. H. Chan, y Y.-L. Huang, «Environmental justice and flood prevention: The moral cost of floodwater redistribution», Landsc. Urban Plan., vol. 189, pp. 36-45, sep. 2019.

[3] J. Batica y P. Gourbesville, «Resilience in Flood Risk Management - A New Communication Tool», Procedia Eng., vol. 154, pp. 811-817, ene. 2016.

[4] L. Bertilsson, K. Wiklund, I. de Moura Tebaldi, O. M. Rezende, A. P. Veról, y M. G. Miguez, «Urban flood resilience - A multicriteria index to integrate flood resilience into urban planning», J. Hydrol., vol. 573, pp. 970-982, jun. 2019.

[5] T. Sarmah y S. Das, «Urban flood mitigation planning for Guwahati: A case of Bharalu basin», J. Environ. Manage., vol. 206, pp. 1155-1165, ene. 2018.

[6] T. Tingsanchali, «Urban flood disaster management», Procedia Eng., vol. 32, pp. 25-37, ene. 2012.

[7] K.-H. Liao, T. A. Le, y K. V. Nguyen, «Urban design principles for flood resilience: Learning from the ecological wisdom of living with floods in the Vietnamese Mekong Delta», Landsc. Urban Plan., vol. 155, pp. 69-78, nov. 2016.

[8] C. Vázquez-González, P. Moreno-Casasola, L. A. Peralta Peláez, R. Monroy, y I. Espejel, «The value of coastal wetland flood prevention lost to urbanization on the coastal plain of the Gulf of Mexico: An analysis of flood damage by hurricane impacts», Int. J. Disaster Risk Reduct., vol. 37, p. 101180, jul. 2019.

[9] R. Cai, H. Zhang, M. Zhang, H. Yang, J. Lyu, y G. Yue, «Development and application of the design principle of fluidization state specification in CFB coal combustion», Fuel Process. Technol., vol. 174, pp. 41-52, jun. 2018.

[10] A. Galderisi y E. Treccozzi, «Green Strategies for Flood Resilient Cities: The Benevento Case Study», Procedia Environ. Sci., vol. 37, pp. 655-666, ene. 2017. 